\title{
Low-dose antibiotics: current status and outlook for the
}

\section{future}

\author{
Joshua D. Nosanchuk ${ }^{1,2 *}$, Jun Lin ${ }^{3}$, Robert P. Hunter ${ }^{4}$ and Rustam I. Aminov ${ }^{5}$ \\ ${ }^{1}$ Department of Medicine, Albert Einstein College of Medicine of Yeshiva University, Bronx, NY, USA \\ ${ }^{2}$ Department of Microbiology and Immunology, Albert Einstein College of Medicine of Yeshiva University, Bronx, NY, USA \\ ${ }^{3}$ Department of Animal Science, The University of Tennessee, Knoxville, TN, USA \\ ${ }^{4}$ Parnell Pharmaceuticals, Overland Park, KS, USA \\ ${ }^{5}$ Section for Bacteriology, Pathology, and Parasitology, National Veterinary Institute, Technical University of Denmark, Frederiksberg, Denmark \\ ${ }^{*}$ Correspondence: josh.nosanchuk@einstein.yu.edu \\ Edited by: \\ Kunihiko Nishino, Osaka University, Japan \\ Reviewed by: \\ Yuji Morita, Aichi Gakuin University, Japan \\ Manuela Coci, National Research Council, Italy
}

Keywords: antibiotics, low dose antibiotics, immunomodulatory effect, environmental impact, growth promotion, feed additives

Antimicrobial therapy is a key factor in our success against pathogens poised to ravage at risk or infected individuals. However, we are currently at a watershed point as we face a growing crisis of antibiotic resistance among diverse pathogens. One area of intense interest is the impact of the application of antibiotics for uses other than the treatment of patients and the association with such utilization with emerging drug resistance. This Research Topic "Low-dose antibiotics: current status and outlook for the future" in Frontiers in Microbiology: Antimicrobials, Resistance, and Chemotherapy details various aspects of the wide ranging effects of antimicrobial therapy from areas such as the regulation of host responses to modulation of bacterial virulence factors to acquisition of antibiotic resistance genes.

A remarkable and often overlooked fundamental of antibiotics is that they have biological activities beyond microbial killing. The host modulatory aspects of macrolides, tetracyclines, and betalactams are reviewed by Aminov (2013a) underscoring how, for example, macrolides such as azithromycin are routinely used for immunomodulation in patients with chronic pulmonary disease rather than for an antimicrobial effect. Azithromycin is also used as a tool by Imperi et al. to detail how non-conventional thinking about regulating virulence factors or modifying host inflammatory cascades are useful to combating major pathogens such as Pseudomonas aeruginosa (Imperi et al., 2014). Along this line, Morita and colleagues carefully detail the pleotropic responses of $P$. aeruginosa to sub-therapeutic levels of several antibacterials and propose avenues to pursue to combat this pathogen, such as developing efflux pump inhibitors (Morita et al., 2014). In their article, Charlebois et al. show Clostridium perfringens biofilm can be regulated by certain antibiotics at low concentrations (Charlebois et al., 2014). For example, low dose bacitracin significantly enhances biofilm formation whereas low dose penicillin reduces biofilm. This work underscores how there are untoward effects that are not predictable when antimicrobials are administered at low concentrations. Providing a view on specific host effector pathways with antimicrobials, Mihu et al. detail how antifungal medications effectively stimulate host responses via engagement with toll-like receptors (Mihu et al., 2014). In light of the expanding difficulties with drug resistance and a lack of therapeutics to combat them, Clark presents a cogent call for pursing $\mathrm{Ca}^{2+}$ modulating strategies where by host $\mathrm{Ca}^{2+}$ homeostasis is modulated to block pathogens from effectively utilizing this essential element (Clark, 2013).

An important focus in this Research Topic is the use of antibiotics as growth enhancers in animals. Sorensen and colleagues provide key insights into the effects of scientific evidence on the policy decisions on the use of low-dose antimicrobials in livestock for growth promotion and disease prevention particularly delineating how data have led to the European Union's ban of low-dose antimicrobials whereas their use in the United States of America remains in flux (Sorensen et al., 2014). The bottom line is that there is an urgent need to develop policy based on well derived data, with this data being easily and widely available to independent parties. The articles by Cheng et al. (2014), Chattopadhyay (2014), and Hao et al. (2014) all further underscore critically important facets of the continued utilization of antibiotics in animal husbandry. Looft and colleagues detail their research on how the use of the in-feed antibiotic carbadox cases dramatic short- and long-term effects on the composition of porcine gut microbiota (Looft et al., 2014). Diarra and Malouin specifically describe the impact of antibiotics in Canadian poultry production and describe the use of alternatives, such as bioactive molecules from cranberries, that should not drive antibiotic resistance (Diarra and Malouin, 2014). Similarly, Rendondo et al. provide thoughtful insights into the use of tannins in lieu of antibiotics for improving health in poultry (Redondo et al., 2014). Lin details that the effective of antibiotics as growth promoters is linked to decreased activities of bile salt hydrolase, which thus makes targeting this enzyme directly a promising method for removing antibiotics for use as growth enhancers (Lin, 2014). 
You and Silbergeld critically discuss the effects of antimicrobials as drivers of resistome expansion (You and Silbergeld, 2014), a major secondary effect due to environmental pollution. The effects of antibiotics permeating our environment are highlighted by Conro and colleagues who present their findings that the presence of antibiotics in aquatic environments can induce co-aggregation of bacterial species as an effective mechanism to combat the effects of the antimicrobials (Corno et al., 2014), which can lead to extensive resistance through the transfer of resistance genes among these aggregated bacteria. It is a small leap for these microbes to then impact humans and other organisms. Aminov provides the example of the rampant use of tetracyclines for non-medical purposes as driving the penetration of tet $(\mathrm{X})$ into pathogenic microbial communities (Aminov, 2013b). Chowdhury and colleagues eloquently discuss the import of surveillance strategies for critically elucidating the emergence of drug resistant pathogens in the context of low-dose antibiotic use in animal husbandry (Roy Chowdhury et al., 2014).

In summary, the articles within this Research Topic serve as a "call to arms" for scientists, policy makers and the public to be increasingly vigilant about the use of antimicrobials, particularly in low-dose or where they can become widespread in the environment, in order to maintain our capacity to effectively care for individuals with infectious diseases. The articles also provide new concepts for approaches for the development of antimicrobials as well as for novel growth enhancers for the use in animal husbandry.

\section{REFERENCES}

Aminov, R. I. (2013a). Biotic acts of antibiotics. Front. Microbiol. 4:241. doi: 10.3389/fmicb.2013.00241

Aminov, R. I. (2013b). Evolution in action: dissemination of tet(X) into pathogenic microbiota. Front. Microbiol. 4:192. doi: 10.3389/fmicb.2013.00192

Charlebois, A., Jacques, M., and Archambault, M. (2014). Biofilm formation of Clostridium perfringens and its exposure to low-dose antimicrobials. Front. Microbiol. 5:183. doi: 10.3389/fmicb.2014.00183

Chattopadhyay, M. K. (2014). Use of antibiotics as feed additives: a burning question. Front. Microbiol. 5:334. doi: 10.3389/fmicb.2014. 00334

Cheng, G., Hao, H., et al. (2014). Antibiotic alternatives: the substitution of antibiotics in animal husbandry? Front. Microbiol. 5:217. doi: 10.3389/fmicb.2014.00217

Clark, K. B. (2013). Biotic activity of $\mathrm{Ca}^{2+}$-modulating nontraditional antimicrobial and -viral agents. Front. Microbiol. 4:381. doi: 10.3389/fmicb.2013.00381
Corno, G., Coci, M., et al. (2014). Antibiotics promote aggregation within aquatic bacterial communities. Front. Microbiol. 5:297. doi: 10.3389/fmicb.2014.00297

Diarra, M. S., and Malouin, F. (2014). Antibiotics in Canadian poultry productions and anticipated alternatives. Front. Microbiol. 5:282. doi: 10.3389/fmicb.2014.00282

Hao, H., Cheng, G., et al. (2014). Benefits and risks of antimicrobial use in foodproducing animals. Front. Microbiol. 5:288. doi: 10.3389/fmicb.2014.00288

Imperi, F., Leoni, L., et al. (2014). Antivirulence activity of azithromycin in Pseudomonas aeruginosa. Front. Microbiol. 5:178. doi: 10.3389/fmicb.2014. 00178

Lin, J. (2014). Antibiotic growth promoters enhance animal production by targeting intestinal bile salt hydrolase and its producers. Front. Microbiol. 5:33. doi: 10.3389/fmicb.2014.00033

Looft, T., Allen, H. K., et al. (2014). Carbadox has both temporary and lasting effects on the swine gut microbiota. Front. Microbiol. 5:276. doi: 10.3389/fmicb.2014.00276

Mihu, M. R., Pattabhi, R., et al. (2014). The impact of antifungals on toll-like receptors. Front. Microbiol. 5:99. doi: 10.3389/fmicb.2014.00099

Morita, Y., Tomida, J., et al. (2014). Responses of Pseudomonas aeruginosa to antimicrobials. Front. Microbiol. 4:422. doi: 10.3389/fmicb.2013.00422

Redondo, L. M., Chacana, P. A., et al. (2014). Perspectives in the use of tannins as alternative to antimicrobial growth promoter factors in poultry. Front. Microbiol. 5:118. doi: 10.3389/fmicb.2014.00118

Roy Chowdhury, P., McKinnon, J., et al. (2014). Genomic interplay in bacterial communities: implications for growth promoting practices in animal husbandry. Front. Microbiol. 5:394. doi: 10.3389/fmicb.2014.00394

Sorensen, A. C., Lawrence, R. S., et al. (2014). Interplay between policy and science regarding low-dose antimicrobial use in livestock. Front. Microbiol. 5:86. doi: 10.3389/fmicb.2014.00086

You, Y., and Silbergeld, E. K. (2014). Learning from agriculture: understanding low-dose antimicrobials as drivers of resistome expansion. Front. Microbiol. 5:284. doi: 10.3389/fmicb.2014.00284

Conflict of Interest Statement: The authors declare that the research was conducted in the absence of any commercial or financial relationships that could be construed as a potential conflict of interest.

Received: 12 August 2014; accepted: 25 August 2014; published online: 10 September 2014.

Citation: Nosanchuk JD, Lin J, Hunter RP and Aminov RI (2014) Low-dose antibiotics: current status and outlook for the future. Front. Microbiol. 5:478. doi: 10.3389/ fmicb.2014.00478

This article was submitted to Antimicrobials, Resistance and Chemotherapy, a section of the journal Frontiers in Microbiology.

Copyright (C) 2014 Nosanchuk, Lin, Hunter and Aminov. This is an open-access article distributed under the terms of the Creative Commons Attribution License (CC BY). The use, distribution or reproduction in other forums is permitted, provided the original author(s) or licensor are credited and that the original publication in this journal is cited, in accordance with accepted academic practice. No use, distribution or reproduction is permitted which does not comply with these terms. 